\title{
AN INTEGRAL REPRESENTATION OF HOLOMORPHIC FUNCTIONS
}

\author{
R. W. HILGER AND J. F. MICHALIČEK
}

\begin{abstract}
Let $K$ be a compact set in the complex plane and let $f$ be a function holomorphic on the complement $\Omega$ of $K$ and vanishing at infinity. We prove that there are finite complex-valued Borel measures $\mu_{m, n}(m$, $n=0,1,2, \ldots ; m+n>1)$ on $K^{2}$ satisfying $\lim _{k \rightarrow \infty}\left(\Sigma_{m+n-k}\left\|\mu_{m, n}\right\|\right)^{1 / k}$ $=0$ so that

$$
f(z)=\sum_{m, n} \int_{K^{2}}\left(z-w_{1}\right)^{-m}\left(z-w_{2}\right)^{-n} d \mu_{m, n}\left(w_{1}, w_{2}\right) \quad(z \in \Omega) .
$$
\end{abstract}

1. Introduction. Let $K$ be a nonempty compact set in the complex plane $\mathbf{C}$ and put $\Omega=\mathrm{C} \backslash K$. The following integral representation of holomorphic functions on $\Omega$ is due to Baernstein [1].

THEOREM 1. If $K$ is locally connected then every holomorphic function $f$ on $\Omega$ vanishing at infinity has a representation

$$
f(z)=\sum_{k=1}^{\infty} \int_{K}(z-w)^{-k} d \mu_{k}(w) \quad(z \in \Omega)
$$

where $\left(\mu_{k}\right)$ are finite complex-valued Borel measures on $K$ satisfying $\lim _{k \rightarrow \infty}\left\|\mu_{k}\right\|^{1 / k}=0$.

This theorem fails if $K$ is not locally connected. Consider e.g. the set $K=\left\{n^{-1} \mid n=1,2, \ldots\right\} \cup\{0\}$ and the function

$$
f(z)=\sum_{n=1}^{\infty}-n^{-2} z^{-2}\left(z-n^{-1}\right)^{-1}
$$

on $\Omega=\mathrm{C} \backslash K$.

In this paper we prove a generalization of Theorem 1 for arbitrary compact sets. We denote by $I$ the set of all pairs $(m, n)$ of nonnegative integers with $m+n \geqslant 1$ and by $K^{k}$ the Cartesian product of $k$ copies of $K$.

THEOREM 2. Let $f$ be a holomorphic function on $\Omega$ vanishing at infinity. There are finite complex-valued Borel measures $\mu_{m, n}$ on $K^{2},(m, n) \in I$, satisfying $\lim _{k \rightarrow \infty}\left(\sum_{m+n=k}\left\|\mu_{m, n}\right\|\right)^{1 / k}=0$ so that

$$
f(z)=\sum_{I} \int_{K^{2}}\left(z-w_{1}\right)^{-m}\left(z-w_{2}\right)^{-n} d \mu_{m, n}\left(w_{1}, w_{2}\right) \quad(z \in \Omega) .
$$

It is in general impossible to characterize a unique representation.

Received by the editors August 6, 1976 and, in revised form, September 10, 1977. AMS (MOS) subject classifications (1970). Primary 30A86; Secondary 46A09. 
COROLlary. Every holomorphic function on $\Omega$ vanishing at infinity has a representation

$$
f(z)=\sum_{k=1}^{\infty} \int_{K^{k}}\left(\left(z-w_{1}\right) \cdots\left(z-w_{k}\right)\right)^{-1} d \mu_{k}\left(w_{1}, \ldots, w_{k}\right) \quad(z \in \Omega)
$$

where $\mu_{k}$ is a finite complex-valued Borel measure on $K^{k}$ and

$$
\lim _{k \rightarrow \infty}\left\|\mu_{k}\right\|^{1 / k}=0 \text {. }
$$

If $K$ consists of one point, we obtain the Laurent expansion of $f$. Regarding the given example we have

$$
\begin{aligned}
f(z) & =\sum_{n=1}^{\infty}-n^{-2} z^{-2}\left(z-n^{-1}\right)^{-1} \\
& =-\int_{K^{3}}\left(\left(z-w_{1}\right)\left(z-w_{2}\right)\left(z-w_{3}\right)\right)^{-1} d \mu_{3}\left(w_{1}, w_{2}, w_{3}\right)
\end{aligned}
$$

where $\mu_{3}$ is a point measure with $\mu_{3}\left\{\left(0,0, n^{-1}\right)\right\}=n^{-2}$ and $z \in \Omega$.

2. Prerequisites. Given a nonempty compact set $S$ in $\mathrm{C}$, we denote by $A(S)$ the Banach algebra of all continuous complex-valued functions on $S$ which are holomorphic on the interior $\stackrel{\circ}{S}$ of $S$ with the norm $\|f\|_{S}=\sup _{s \in S}|f(s)|$ $(f \in A(S))$.

Let $H(K)$ be the algebra of all germs of holomorphic functions on $K$. Consider a base $\left\{S_{\lambda} \mid \lambda=1,2, \ldots\right\}$ of compact neighbourhoods of $K$ whose boundaries $\partial S_{\lambda}$ are finite unions of rectifiable Jordan curves. Suppose additionally that every component of $S_{\lambda}$ intersects $K$. With the inductive topology with respect to the natural mappings $A\left(S_{\lambda}\right) \rightarrow H(K), H(K)$ is a topological vector space. This topology is independent of the base $\left\{S_{\lambda}\right\}$.

We denote by $H(\Omega)$ the algebra of all holomorphic functions on $\Omega$ vanishing at infinity. Consider a sequence $\left(X_{\lambda}\right)_{\lambda=1,2, \ldots}$ of compact sets $X_{\lambda} \subset \Omega$ with $X_{\lambda} \subset \dot{X}_{\lambda+1}$ and $\Omega=\cup \lambda_{\lambda=1}^{\infty} X_{\lambda}$. With the projective topology with respect to the natural mappings $H(\Omega) \rightarrow A\left(X_{\lambda}\right), H(\Omega)$ is a topological vector space. This topology is independent of $\left(X_{\lambda}\right)$.

Let us list some well-known properties of $H(K)$ and $H(\Omega)([2],[3]) . H(K)$ and $H(\Omega)$ are Montel spaces and therefore reflexive. $H(K)$ and $H(\Omega)$ are strong duals with respect to the following bilinear form. Consider $f \in H(K)$ and $g \in H(\Omega)$. Then there exists a $\lambda$ with $f \in A\left(S_{\lambda}\right)$.

$$
\langle f, g\rangle=\frac{1}{2 \pi i} \int_{\partial S_{\lambda}} f(z) g(z) d z
$$

defines a continuous bilinear form on $H(K) \times H(\Omega)$.

Let $L_{\nu}(\nu=1,2, \ldots)$ be the Banach space of all families $\left(\varphi_{m, n}\right)_{(m, n) \in I}$ of continuous functions $\varphi_{m, n}$ on $K^{2}$ satisfying

$$
\left\|\left(\varphi_{m, n}\right)\right\|_{\nu}=\sup _{m, n}\left(\sup _{K^{2}}\left|\varphi_{m, n}\right|\right) \nu^{-(m+n)}<\infty
$$


and

$$
\lim _{m+n \rightarrow \infty}\left(\sup _{K^{2}}\left|\varphi_{m, n}\right| \nu^{-(m+n)}\right)=0 .
$$

$L=\cup_{\nu=1}^{\infty} L_{\nu}$ is a topological vector space with the inductive topology with respect to the inclusions $L_{\nu} \rightarrow L$.

Let $M_{\nu}$ be the Banach space of all families $\left(\mu_{m, n}\right)_{(m, n) \in I}$ of finite complex valued Borel measures on $K^{2}$ satisfying

$$
\left\|\left(\mu_{m, n}\right)\right\|_{\nu}=\sum_{m, n}\left\|\mu_{m, n}\right\| \nu^{m+n}<\infty
$$

$M=\cap_{\nu=1}^{\infty} M_{\nu}$ is a topological vector space with the projective topology with respect to the inclusions $M \rightarrow M_{\nu} . M$ is the set of all families $\left(\mu_{n, m}\right)_{(m, n) \in I}$ satisfying $\lim _{k \rightarrow \infty}\left(\sum_{m+n=k}\left\|\mu_{m, n}\right\|\right)^{1 / k}=0$.

Consider the bilinear form on $L_{\nu} \times M_{\nu}$ defined by

$$
\left\langle\left(\varphi_{m, n}\right),\left(\mu_{m, n}\right)\right\rangle=\sum_{I} \int_{K^{2}} \varphi_{m, n}\left(w_{1}, w_{2}\right) d \mu_{m, n}\left(w_{1}, w_{2}\right) .
$$

Since

$$
\left|\left\langle\left(\varphi_{m, n}\right),\left(\mu_{m, n}\right)\right\rangle\right| \leqslant\left\|\left(\varphi_{m, n}\right)\right\|_{\nu}\left\|\left(\mu_{m, n}\right)\right\|_{\nu} .
$$

Riesz' theorem shows that $M_{\nu}$ is the strong dual space of $L_{\nu}$. Hence the strong dual space of $L$ is isomorphic to $M$ [3].

$H(K)$ and $L$ are bornological $(D F)$-spaces [3, p. 406], $H(\Omega)$ and $M$ are $(F)$-spaces. $H(K)$ and $H(\Omega)$ are Montel spaces [2].

Proposition 1 [3]. Let $E, F$ be locally convex spaces, $E$ bornological; a linear mapping $T: E \rightarrow F$ is continuous if and only if $T(B) \subset F$ is bounded whenever $B \subset E$ is bounded.

Proposition 2 [1]. Let $E$ be a Montel space, $F$ a $(D F)$-space and $T: E \rightarrow F$ a continuous linear mapping. If $T^{-1}(B)$ is bounded in $E$ for all bounded sets $B \subset F$, then $T: E \rightarrow T(E)$ is an isomorphism of topological vector spaces.

3. Embedding of $H(K)$ into $L$. Consider the linear mapping $T: H(K) \rightarrow L$ defined by

$$
(T f)_{m, n}\left(w_{1}, w_{2}\right)=\left\langle f,\left(z-w_{1}\right)^{-m}\left(z-w_{2}\right)^{-n}\right\rangle \quad(f \in H(K)) .
$$

THEOREM 3. $T: H(K) \rightarrow T(H(K))$ is an isomorphism of topological vector spaces.

Proof. Let $B$ be a bounded set in $H(K)$. Then $B$ is bounded in $A\left(S_{\lambda}\right)$ for some $\lambda$. For $f \in B$ we have

$$
\begin{aligned}
\left|(T f)_{m, n}\left(w_{1}, w_{2}\right)\right| & =\left|\frac{1}{2 \pi i} \int_{\partial S_{\lambda}} f(z)\left(z-w_{1}\right)^{-m}\left(z-w_{2}\right)^{-n} d z\right| \\
& \leqslant(2 \pi)^{-1}\left|\partial S_{\lambda}\right|\|f\|_{S_{\lambda}} \delta^{-(m+n)},
\end{aligned}
$$

where $\delta$ is the distance between $K$ and $\partial S_{\lambda}$ and $\left|\partial S_{\lambda}\right|$ is the length of $\partial S_{\lambda}$. If 
we choose $\nu>\delta^{-1}+1$ we conclude $T f \in L_{\nu}$

$$
\begin{aligned}
\lim _{m+n \rightarrow \infty}\left(\sup _{K^{2}}\left|\varphi_{m, n}\right| \nu^{-(m+n)}\right) & \leqslant \lim _{m+n \rightarrow \infty}(2 \pi)^{-1}\left|\partial S_{\lambda}\right|\|f\| S_{\lambda} \delta^{-(m+n)} \nu^{-(m+n)} \\
& <\lim _{m+n \rightarrow \infty}(\nu-1)^{(m+n)} \nu^{-(m+n)}=0
\end{aligned}
$$

and that

$$
\begin{aligned}
\|T f\|_{\nu} & =\sup _{m, n}\left(\sup _{K^{2}}\left|(T f)_{m, n}\right| \nu^{-(m+n)}\right) \\
& \leqslant \sup _{m, n}(2 \pi)^{-1}\left|\partial S_{\lambda}\right|\|f\| S_{\lambda}(\delta \cdot \nu)^{-(m+n)} \\
& \leqslant(2 \pi)^{-1}\left|\partial S_{\lambda}\right|\|f\| s_{\lambda} .
\end{aligned}
$$

Thus $T(B)$ is bounded in $L_{\nu}$ and therefore $T(B)$ is bounded in $L$. The continuity of $T$ then follows from Proposition 1.

We now apply Proposition 2 to prove that $T: H(K) \rightarrow T(H(K))$ is an isomorphism. Let $B$ be a bounded set in $L$. Then $B$ is bounded in $L_{\nu}$ for some $\nu$. At first we show that $T^{-1}(B) \subseteq A\left(S_{\lambda}\right)$ for some $\lambda$. Suppose the contrary: for every positive $\varepsilon$ there exists a germ $f \in T^{-1}(B)$ which has no holomorphic continuation on $U_{\varepsilon / 2}(K)$, the $\varepsilon / 2$-neighbourhood of $K$. We choose $\varepsilon<(4 v)^{-1}$. Since $(j !)^{-1} f^{(j)}(w)=(T f)_{1, j}(w, w) \quad(w \in K)$, the power series $\sum_{j=0}^{\infty}\left(f^{(j)}(w) / j !\right)(z-w)^{j}$ converges for $|z-w|<\nu^{-1}$. According to our supposition there are points $w_{1}, w_{2} \in K,\left|w_{1}-w_{2}\right|<\varepsilon$, so that the power series of $f$ in powers of $z-w_{1}$ and powers of $z-w_{2}$ are not continuations of each other. Let $R_{2}$ be a disc with center $w_{2}$ and radius $r_{2}=(2 v)^{-1}$. Let $R_{1}$ be a disc with center $w_{1}$ and radius $r_{1}<\left|w_{1}-w_{2}\right|$. The Taylor series of $f$ in powers of $z-w_{2}$ defines a holomorphic function $g$ on $R_{1}$. If $D_{1}$ and $D_{2}$ are small discs centred at $w_{1}, w_{2}$ then

$$
(T f)_{m, n}\left(w_{1}, w_{2}\right)=\frac{1}{2 \pi i} \int_{\partial D_{1} \cup \partial D_{2}} f(z)\left(z-w_{1}\right)^{-m}\left(z-w_{2}\right)^{-n} d z .
$$

In the second integral we expand $f$ in powers of $z-w_{2}$ and use

$$
\begin{aligned}
\int_{\partial D_{2}}\left(z-w_{1}\right)^{-m}\left(z-w_{2}\right)^{-n} d z= & \int_{\partial R_{2}}\left(z-w_{1}\right)^{-m}\left(z-w_{2}\right)^{-n} d z \\
& -\int_{\partial R_{1}}\left(z-w_{1}\right)^{-m}\left(z-w_{2}\right)^{-n} d z
\end{aligned}
$$

from which is easily deduced the equation

$$
\begin{aligned}
(T f)_{m, n}\left(w_{1}, w_{2}\right)= & \frac{1}{2 \pi i} \int_{\partial R_{1}} \sum_{j=0}^{\infty} \frac{1}{j !}\left(f^{(j)}-g^{(j)}\right)\left(w_{1}\right)\left(z-w_{1}\right)^{j-m}\left(z-w_{2}\right)^{-n} d z \\
& +\frac{1}{2 \pi i} \int_{\partial R_{2}} \sum_{j=0}^{\infty} \frac{1}{j !} f^{(j)}\left(w_{2}\right)\left(z-w_{1}\right)^{-m}\left(z-w_{2}\right)^{j-n} d z .
\end{aligned}
$$

Let $m_{0}$ be the lowest index such that $f^{\left(m_{0}\right)}\left(w_{1}\right) \neq g^{\left(m_{0}\right)}\left(w_{1}\right)$. We fix $m=m_{0}+1$ 
and obtain

$$
\begin{aligned}
(T f)_{m, n}\left(w_{1}, w_{2}\right)= & \frac{1}{m_{0} !}\left(f^{\left(m_{0}\right)}-g^{\left(m_{0}\right)}\right)\left(w_{1}\right)\left(w_{1}-w_{2}\right)^{-n} \\
& +\frac{1}{2 \pi i} \int_{\partial R_{2}} \sum_{j=0}^{\infty}(T f)_{1, j}\left(w_{2}, w_{2}\right)\left(z-w_{1}\right)^{-m}\left(z-w_{2}\right)^{j-n} d z .
\end{aligned}
$$

Note that if $z \in \partial R_{2}$, then $\left|z-w_{1}\right|>(4 \nu)^{-1},\left|z-w_{2}\right|=(2 v)^{-1}$, and $\mid w_{1}-$ $w_{2} \mid<(4 \nu)^{-1}$. Hence

$$
\begin{aligned}
\left|(T f)_{m, n}\left(w_{1}, w_{2}\right)\right| \geqslant & \frac{1}{m_{0} !}\left|f^{\left(m_{0}\right)}\left(w_{1}\right)-g^{\left(m_{0}\right)}\left(w_{1}\right)\right| 4^{n} \nu^{n} \\
& -\|T f\|_{\nu} 2^{n+2 m_{0}+2} \nu^{n+m_{0}+1}
\end{aligned}
$$

and we conclude $\|T f\|_{\nu}=\infty$ which is a contradiction to $T f \in L_{\nu}$.

Finally we show that $T^{-1}(B)$ is bounded in $A\left(S_{\lambda}\right)$. Suppose $S_{\lambda} \subseteq$ $U_{1 / 2 \nu}(K)$. For every $z \in S_{\lambda}$ there exists a $w \in K$ so that $|z-w|<(2 \nu)^{-1}$. Then for all $f \in T^{-1}(B)$ we have

$$
f(z)=\sum_{j=0}^{\infty}(T f)_{1, j}(w, w)(z-w)^{j} .
$$

Hence

$$
\|f\|_{S_{\lambda}} \leqslant \sup _{z \in S_{\lambda}} \sum_{j=0}^{\infty}\left|(T f)_{1, j}(w, w)\right||z-w|^{j} \leqslant \nu\|T f\|_{\nu} .
$$

Thus $T^{-1}(B)$ is bounded in $A\left(S_{\lambda}\right)$ and therefore $T^{-1}(B)$ is bounded in $H(K)$. Proposition 2 completes the proof.

COROLlaRY. The adjoint mapping $T^{*}: L^{*}=M \rightarrow H(K)^{*}=H(\Omega)$ is surjective.

Proof of Theorem 2. Suppose $f \in H(\Omega)$. The last corollary shows the existence of $\left(\mu_{n, m}\right) \in M$ such that $T^{*}\left(\mu_{m, n}\right)=f$. Let

$$
\varphi(z)=\sum_{I} \int_{K^{2}}\left(z-w_{1}\right)^{-m}\left(z-w_{2}\right)^{-n} d \mu_{m, n}\left(w_{1}, w_{2}\right) .
$$

Then $\varphi \in H(\Omega)$ and, by use of Fubini's Theorem, we deduce $\langle h, f\rangle=\langle T h$, $\left.\left(\mu_{m, n}\right)\right\rangle=\langle h, \varphi\rangle$, for all $h \in H(K)$. It follows that $f=\varphi$.

\section{REFERENCES}

1. A. Baernstein, Representation of holomorphic functions by boundary integrals, Trans. Amer. Math. Soc. 160 (1971), 27-37.

2. G. Köthe, Dualität in der Funktionentheorie, J. Reine Angew. Math. 191 (1953), 30-49.

3. , Topological vector spaces. I, Springer-Verlag, Berlin, 1969.

University of Hamburg, 2 Hamburg 13, Federal Republic of Germany 